Note

\section{Precursors to Nitrosopyrrolidine and Nitrosopiperidine in Black Pepper Treated with Nitrous Acid}

\author{
Masamichi NaKamura, ${ }^{*}$ Kuni KatoH* \\ and Toshiharu KAWABATA** \\ * Department of Food and Drug Science, \\ Kanagawa Prefectural Public Health Laboratory, \\ 52-2 Nakao-cho, Asahi-ku, \\ Yokohama, 241, Japan \\ **Department of Biomedical Research on Food, \\ National Institute of Health of Japan, \\ 2-10-35 Kamiosaki, Shinagawa-ku, \\ Tokyo 141, Japan
}

Received March 3, 1980

Much attention has been paid to the possible formation of carcinogenic $\mathrm{N}$-nitrosamine in food, especially nitritetreated meat and fish products. ${ }^{1 \sim 3)}$ Sen $e t$ al $^{\left({ }^{4}\right)}$ reported that fairly high levels of nitrosopyrrolidine (NPYR) were detected in mettwurst sausage produced by certain Canadian farms. They disclosed that black pepper and paprika added to meat mixtures can react with nitrite to form nitrosopiperidine (NPIP) and NPYR, and they suggested that NPIP and NPYR may be formed from piperidine, pyrrolidine or their precursors in such spices as black pepper or paprika. In the present study, we searched for the precursors responsible for the formation of NPYR and NPIP in a benzene extract of black pepper.

Commercial ground black pepper (Piper nigrum L.) imported from Malaysia was used. Piperine(1-piperoyl piperidine) purchased from Aldrich Chemical Co. Inc. was purified by thin-layer chromatography (TLC) and recrystallized three times from ethanol saturated with $n$ hexane. Pyrroperine(1-piperoyl pyrrolidine) was kindly provided by Dr. K. Mori of Nippon Shinyaku Co. and used without further purification. NPIP and NPYR were synthesized from piperidine and pyrrolidine according to the method of Uno and Yamamoto. ${ }^{5)}$ Other reagents were of analytical-reagent grade.

Nitrosation reaction and determination of nitrosamines: The reactants were dissolved in water and the $\mathrm{pH}$ of the solution was adjusted to 3.0 with $\mathrm{HClO}_{4}$. To each of these solutions was added nitrite preadjusted to $\mathrm{pH} 3.0$ with $\mathrm{HClO}_{4}$ and then the mixtures were kept at $37^{\circ} \mathrm{C}$ for 3 hours. The nitrosation reaction was stopped by adding $30 \% \mathrm{NaOH}$ solution. Then the nitrosamines formed were extracted with $\mathrm{CH}_{2} \mathrm{Cl}_{2}$ in the presence of an excess amount of $\mathrm{NaCl}$. Aliquots of the $\mathrm{CH}_{2} \mathrm{Cl}_{2}$ extract were investigated by gas chromatography (GC) and by gas chromatographic-mass spectrometric (GC-MS) analysis. GC and

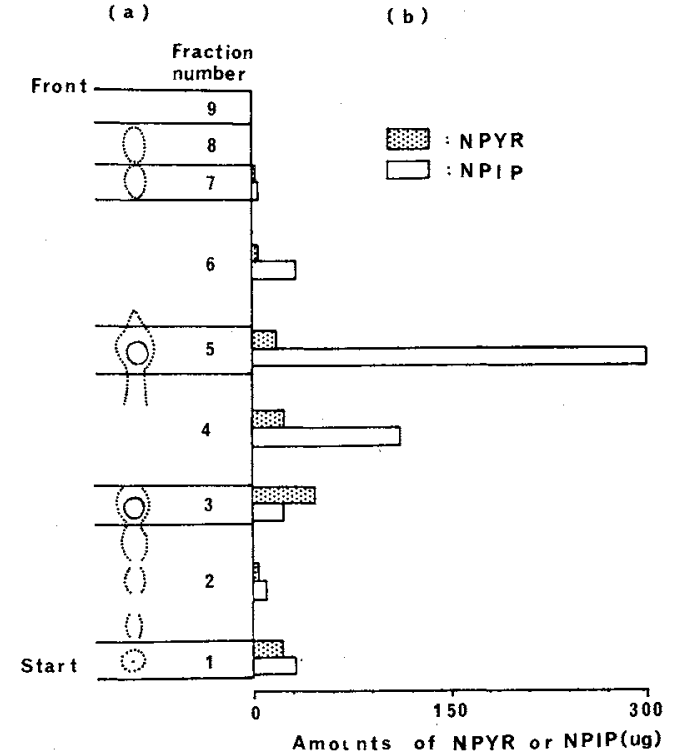

FIG. 1. Thin-layer Chromatogram of the Benzene Extract of Black Pepper (a) and the Amounts of NPIP and NPYR Produced after Nitrosation of the Extract from Each Fraction.

(a): One gram of black pepper was extracted with benzene and the extract was evaporated under reduced pressure. Then the extract was spotted on a silica gel plate (Merck No. 574) and this was developed with a solvent mixture of ethyl acetate-carbon tetrachloride $(1: 1)$ in a darkroom. After being developed, fuorescent spots on the plate were detected under UV light irradiation $(365 \mathrm{~nm}$, "Super Light," Nikko Sekiei Works). The intensity of dots on TLC, on the circular and semi-circular images show the degree of fluorescence. Therefore, unbroken lines (as in 3 and 5) mean strong fluorescence, while dotted lines mean weak fluorescence.

(b): Each fraction of the nine parts of TLC was extracted with ethanol and the extract was evaporated and treated with ten grams of $\mathrm{NaNO}_{2}$ as described in the text. Reaction conditions were as follows; $\mathrm{pH} 3.0$ (adjusted with $\mathrm{HClO}_{4}$ ) $37^{\circ} \mathrm{C}, 3 \mathrm{hr}$. Then NPIP or NPYR produced after nitrosation was extracted with $\mathrm{CH}_{2} \mathrm{Cl}_{2}$. An aliquot of the $\mathrm{CH}_{2} \mathrm{Cl}_{2}$ extract was analyzed by $\mathrm{GC}$. $\mathrm{GC}$ conditions were as follows: column, 15\% PEG $20 \mathrm{M}$ on Chromosorb WAW (60 80 mesh); column temp., $170^{\circ} \mathrm{C}$; detector, AFID.

GC-MS analyses were conducted according to Kawabata et al.'s method. ${ }^{6}$ As shown in Fig. 1, the thin-layer chromatogram of the benzene extract of one gram of black pepper was divided into nine parts under UV light $(365 \mathrm{~nm})$ irradiation. Each portion was scraped off, extracted with ethanol, and the solvent completely removed under reducing pressure. The residue was 
nitrosated with ten grams of $\mathrm{NaNO}_{2}$ under acidic conditions and the resultant nitrosamine was extracted with $\mathrm{CH}_{2} \mathrm{Cl}_{2}$, followed by the $\mathrm{GC}$ and $\mathrm{GC}-\mathrm{MS}$ analyses described above. The largest amounts of NPYR and NPIP were detected in fractions 3 and 5, respectively. Small amounts of both NPIP and NPYR were detected in fraction 1 at the starting point. However, this spot seemed to be different from other fractions, because fraction 1 was positive to the ninhydrin reaction but fractions 3 and 5 were negative to the ninhydrin reaction. Fractions 3 and 5 coincided with authentic pyrroperine and piperine on TLC, respectively. The substances extracted with ethanol from fractions 3 and 5 were redeveloped separately on TLC plates, and portions corresponding to piperine and pyrroperine were scraped off and extracted with ethanol. The residue after the removal of ethanol was recrystallized three times from ethanol saturated $n$-hexane. Melting points of isolated materials corresponded to those of pyrroperine and piperine $\left(145^{\circ}\right.$ and $\left.130^{\circ} \mathrm{C}\right)$. And depression of melting points of these compounds was not observed on admixture with each authentic compound. The GC-MS fragments of these compounds were $\mathrm{m} / \mathrm{z}$ (Relative intensity in \%), $271(68), 201(100), 173(40), 143$ (23), $115(40), 70(11)$ and $55(8)$, and $m / z, 285(86), 201$ $(100), 173(38), 143(24), 115(48), 84(31)$ and $69(6)$. Mass spectrometric analysis of these substances revealed that the main component in fraction 3 was pyrroperine, and that in fraction 5 was piperine. The contents of piperine and pyrroperine in the black pepper sample were $4.6 \%$ and $0.1 \%$, respectively. Piperine and pyrroperine were determined according to Mori et al.'s method, ${ }^{7}$ i.e., the extract was separated by TLC and the parts corresponding to authentic compounds were extracted with ethanol and measured at $343 \mathrm{~nm}^{7}{ }^{7}$ Fraction 1 scraped off was extracted with $0.5 \mathrm{~N} \mathrm{HCl}$. After the extract was washed three times with benzene, the solution was alkalified with $\mathrm{NaOH}$ and extracted three times with diethylether. The combined ether extract was further extracted with $0.5 \mathrm{~N}$ $\mathrm{HCl}$. The extract was nitrosated in the same manner as described above. The GC-MS fragments of the compounds were $m / z, 114$ (100), 55 (77), 42 (88), 30 (39), $27(30)$ and $15(10)$, and $m / z, 100(100), 43(38), 41(81), 30$ $(26), 28(78), 27(32)$ and $15(15)$. These two compounds were identified as NPIP and NPYR, respectively. The results suggest the possible presence of small amounts of both pyrrolidine and piperidine in the benzene extract. The contents of piperidine and pyrrolidine in the benzene extract of black pepper were $18 \mathrm{ppm}$ and $5 \mathrm{ppm}$, respectively. The yield of NPIP and NPYR from the secondary amines, piperidine and pyrrolidine, was larger than the yield of NPIP and NPYR from piperine and pyrroperine, kinds of tertiary amines. At the higher reaction temperature larger amounts of nitrosamines were formed. The optimum $\mathrm{pH}$ for nitrosation of these compounds was found to be about 3.0. On the other hand, secondary amine contents in the benzene extract were much lower than tertiary amines. Therefore the amounts of nitrosamine formed from tertiary amines were larger than those of secondary amines. As shown in Fig. 1, the amounts of NPIP and NPYR formed from the benzene extract of one gram of black pepper were $522 \mathrm{ppm}$ and $129 \mathrm{ppm}$, respectively. However, the amounts of nitrosamine formed from piperine, pyrroperine, piperidine and pyrrolidine were about ten per cent of the nitrosamine formed from the benzene extract of pepper. This implies that nitrosamines formed from the benzene soluble fraction of pepper originated from other components than the four compounds described above.

Table I. Reaction of Prperine, Pyrroperine, Piperidine AND Pyrrolidine With Nitrite under Acidic Conditions

\begin{tabular}{|c|c|c|c|c|c|c|c|c|c|}
\hline \multirow{2}{*}{ Compound } & \multicolumn{7}{|c|}{ Reaction conditions } & \multicolumn{2}{|c|}{$\begin{array}{c}\text { Yield of } \\
\text { nitrosamines }(\%)\end{array}$} \\
\hline & Weight (mg) & $\begin{array}{l}\mathrm{NaNO}_{2} \\
(\mathrm{mg})\end{array}$ & Acid & $\begin{array}{l}\text { Vol } \\
(\mathrm{ml})\end{array}$ & $\mathrm{pH}$ & $\begin{array}{c}\text { Temp. } \\
\left({ }^{\circ} \mathrm{C}\right)\end{array}$ & $\begin{array}{l}\text { Time } \\
\text { (hr) }\end{array}$ & NPIP & NPYR \\
\hline \multirow{4}{*}{ Piperine } & $1\left(3.5 \times 10^{-3} \mathrm{~mm}\right)$ & 100 & $\mathrm{HClO}_{4}$ & 10 & 3.0 & 37 & 3 & 0.10 & \\
\hline & 10 & 100 & $\mathrm{HClO}_{4}$ & 10 & 1.0 & 100 & 2 & 0.88 & \\
\hline & 10 & 100 & $\mathrm{HClO}_{4}$ & 10 & 3.0 & 100 & 2 & 2.36 & \\
\hline & 10 & 100 & $\mathrm{HClO}_{4}$ & 10 & 5.0 & 100 & 2 & 0.60 & \\
\hline \multirow{4}{*}{ Pyrroperine } & $1\left(3.7 \times 10^{-3} \mathrm{~mm}\right)$ & 100 & $\mathrm{HClO}_{4}$ & 10 & 3.0 & 37 & 3 & & 0.14 \\
\hline & 0.5 & 50 & $\mathrm{HClO}_{4}$ & 5 & 1.0 & 100 & 2 & & 0.33 \\
\hline & 0.5 & 50 & $\mathrm{HClO}_{4}$ & 5 & 3.0 & 100 & 2 & & 2.20 \\
\hline & 0.5 & 50 & $\mathrm{HClO}_{4}$ & 5 & 5.0 & 100 & 2 & & 0.25 \\
\hline Piperidine & $0.30\left(3.5 \times 10^{-3} \mathrm{mM}\right)$ & 100 & $\mathrm{HClO}_{4}$ & 10 & 3.0 & 37 & 3 & 1.64 & \\
\hline Pyrrolidine & $0.26\left(3.7 \times 10^{-3} \mathrm{~mm}\right)$ & 100 & $\mathrm{HClO}_{4}$ & 10 & 3.0 & 37 & 3 & & 1.51 \\
\hline
\end{tabular}


Acknowledgments. We wish to express our thanks to Dr. K. Mori, Director of the Food Technology Institute of Nippon Shinyaku Co. for providing synthetic pyrroperine, and to Dr. N. Akimori of Hitachi Seisakusho Co. for carrying out the mass spectrometric analysis.

\section{REFERENCES}

1) F. Ender and L. Ceh, Food Cosmet. Toxicol., 6, 569 (1968).

2) N. P. Sen, Food Cosmet. Toxicol., 10, 219 (1972).
3) T. Fazio, J. V. Damico, J. H. Howard, R. H. White and J. O. Watts, J. Agric. Food Chem., 19, 250 (1971).

4) N. P. Sen, W. F. Miles, B. Donaldson, T. Panalaks and J. R. Iyengar, Nature, 245, 104 (1973).

5) T. Uno and M. Yamamoto, Japan Analyst, 15, 958 (1966).

6) T. Kawabata, M. Matsui, T. Ishibashi and M. Nakamura, Japan Analyst, 21, 1327 (1973); T. Kawabata, M. Nakamura, M. Matsui and T. Ishibashi, Bull. Japan Soc. Sci. Fish, 40, 79 (1974).

7) K. Mori, Y. Yamamoto and S. Komai, Nippon Shokuhin Kogyo Gakkai-Shi, 21, 466 (1974). 